\title{
Highly magneto-responsive elastomeric films by a two-step fabrication process
}

Sophie Marchi, ${ }^{*}$ Alberto Casu, ${ }^{b-c}$ Franco Bertora, ${ }^{d}$ Athanassia Athanassiou, ${ }^{a}$ Despina Fragouli. ${ }^{* a}$

${ }^{a}$ Smart Materials, Istituto Italiano di Tecnologia, Via Morego 30, 16163 Genova, Italy.

${ }^{b}$ Nanochemistry, Istituto Italiano di Tecnologia, Via Morego 30, 16163 Genova, Italy.

${ }^{c}$ Biological and Environmental Sciences and Engineering Division, King Abdullah University for Science and Technology, Thuwal 23955-6900 (Kingdom of Saudi Arabia).

${ }^{\mathrm{d}}$ Robotics Brain and Cognitive Science, Istituto Italiano di Tecnologia, Via Morego 30, 16163 Genova, Italy.

KEYWORDS: PDMS, microparticles, magnetic assembly, ferromagnetism, magnetic actuation.

ABSTRACT: An innovative method for the preparation of elastomeric magnetic films with increased magneto-responsivity is presented. Polymeric films containing aligned magnetic microchains throughout their thickness are formed upon the magnetophoretic transport and assembly of microparticles during the polymer curing. The obtained films are subsequently magnetized at a high magnetic field of $3 \mathrm{~T}$ directed parallel to the microchains orientation. We prove that the combination of both, alignment of the particles along a favorable direction during 
curing, and subsequent magnetization of the solid films induces an impressive increase of the films' deflection. Specifically, the displacements reach few millimeters, up to 85 times higher compared to the non-treated films with the same particles concentration. Such process can improve the performance of the magnetic films without increasing the amount of magnetic fillers, thus without compromising the mechanical properties of the resulting composites. The proposed method can be used for the fabrication of magnetic films suitable as components in systems where high displacements at relatively low magnetic fields are required, such as sensors, drug delivery or microfluidic systems, especially where remote control of valves is requested to achieve appropriate flow and mixing of liquids.

\section{Introduction:}

Polymeric membrane actuators have been extensively employed as components in diverse technological systems such as micropumps, or valves in microfluidic devices. ${ }^{1-15}$ For these purposes, various actuation principles have been adopted including electrostatic, ${ }^{2,3,6}$ thermal $^{4,14}$ or piezoelectric. ${ }^{1,7}$ However, these methods present diverse limitations, making difficult their direct utilization in the final devices. In particular, in the case of the electrostatic actuation it is generally required a high driving voltage,${ }^{16,17}$ while the response speed of thermally actuated membranes is usually slow. ${ }^{18}$ Lastly, in a lot of cases, piezoelectric polymeric films exhibit a creep effect ${ }^{19}$ meaning that the material continues to expand even after the removal of the actuation stimulus prohibiting thus the immediate return to its initial state.

The magnetic actuation results to be an interesting alternative to the abovementioned principles, due to the lack of electrical connections, the possibility to be manipulated at a large distance and the fast response to the external magnetic field. ${ }^{9,11,13}$ The first magnetic polymeric actuators, made from a mixture of magnetic particles with rigid polymeric matrices, were mainly 
used as permanent magnets, magnetic cores or connecting elements. ${ }^{20}$ These classical actuators presented low flexibility and their size or shape remained unaffected under the effect of the magnetic field, limiting their range of applications.

Alternatively, the new generation of magnetic elastomers, which consists of magnetic nano or microparticles (such as iron, $\mathrm{Fe}_{3} \mathrm{O}_{4}$ or carbonyl iron) dispersed in highly elastic polymeric matrices, can achieve large and non-homogeneous deformation together with a fast response to an external magnetic field. ${ }^{11,12,21-25}$ Therefore, this class of magnetic materials opened new opportunities for their utilization in various applications where soft actuators are required such as microfluidic systems or micropumps. ${ }^{12,21}$ Indeed, the low elastic modulus of the polymeric matrix allows a direct transfer to the polymeric chains of the forces acting on the magnetic particles under the external field, thus resulting in rapid actuation of flat membranes. However, in order to obtain high deflection values, relatively high magnetic fields should be applied (e.g. under an external magnetic field of $417 \mathrm{mT}$, a deflection of $125 \mu \mathrm{m}$ is observed for a film of 4 $\mathrm{mm}$ in diameter) ${ }^{11}$ or high diameters should be employed (e.g. $125 \mu \mathrm{m}$ deflection under $18 \mathrm{mT}$ magnetic field for a film of $50 \mathrm{~mm}$ in diameter). ${ }^{26}$ This phenomenon is the main drawback for their direct utilization in many applications.

One possibility to increase the magnetic response, and therefore the deflection of these systems in lower fields, is the use of magnetic particles with higher performance, such as the anisotropic rare earth ferromagnetic powders like $\mathrm{Nd}_{2} \mathrm{Fe}_{14} \mathrm{~B}$ alloys. ${ }^{9,27}$ Such microparticles present exceptional magnetic properties ${ }^{27,28}$ and can be permanently magnetized. Alternatively, the magnetic response of the polymeric magnetic films can be sufficiently improved arranging the particles in a preferential direction throughout the volume of the polymeric matrix. In this way, the magnetic properties of the films become anisotropic. Previous works have evidenced the 
possibility to align magnetic particles within thin plastic films by magnetophoretic transport and assembly of the dispersed magnetic particles. ${ }^{29-34}$ The dimensions of the wires are strictly dependent on the strength of the applied magnetic field, the duration of its application and the curing or evaporation rate of the solvent if present. Their magnetization has a strong anisotropy with a higher value towards the direction of the assembled chains ${ }^{29,30,32}$ allowing the formation of functional magnetic films.

However, to the best of our knowledge, only few studies point on the deflection enhancement of polymeric films due to the magnetophoretic transport and assembly of magnetic particles in a polymer film. ${ }^{35}$ Most importantly, the combination of the alignment of anisotropic ferromagnetic particles in a polymeric film and the subsequent magnetization of the solid composite results in an interesting way to highly increase the magneto-responsivity of the films. Therefore, herein we report the preparation of highly magneto-responsive elastomeric composite films by using microparticles of $\mathrm{Nd}_{2} \mathrm{Fe}_{14} \mathrm{~B}$ alloy as fillers assembled preferentially in a defined direction. We show that the magnetophoretic transport and assembly of the particles, which results in the formation of microchains of assembled particles throughout the volume of the film, in combination with the subsequent magnetization of the solid composite lead to an impressive increase of the films' deflection. Specifically, under low magnetic fields, the values are up to 85 times higher compared to those of non-treated composites with the same content of particles. Therefore, such type of process improves the performance of the magnetic films without increasing the amount of magnetic fillers in the polymer matrix, which is kept to relatively low concentrations in order to preserve the pristine mechanical characteristics of the elastomeric matrix.

\section{Experimental Section:}


2.1. Materials. The silicon elastomer Ecoflex 10 from Smooth-on was used as received. It consists of two components: a prepolymer and a curing agent (Mixing ratio 1:1). Anisotropic ferromagnetic particles MQA-36-18 based on a $\mathrm{Nd}_{2} \mathrm{Fe}_{14} \mathrm{~B}$ alloy (PrNdFeCoBDyGa) with average size $\mathrm{D}_{50}<105 \mu \mathrm{m}$ were a kind offer from Magnequench GmbH (Molycorp Company, Germany). The magnetic properties of the particles are the following: Remanence: $\mathrm{B}_{\mathrm{r}}=1240 \mathrm{mT}$, Intrinsic Coercivity: $\mathrm{H}_{\mathrm{ci}}=18.0 \mathrm{kOe}$, and Energy Product $(\mathrm{BH})_{\max }=36$ MGOe. The morphology of the particles and their composition are presented in Figure S1 of the Supporting Information file.

2.2. Samples preparation. The magnetic particles are manually mixed with the prepolymer (10 to $50 \mathrm{wt} \%$ with respect to the final composite) and sonicated by ultrasonic bath for few minutes at $59 \mathrm{kHz}$ in order to obtain the optimum dispersion. Subsequently, the curing agent is added (weight ratio 1:1 prepolymer/curing agent) and, after manual mixing for few seconds, the final solution is poured onto a Teflon substrate, spin-coated at $500 \mathrm{rpm}$ for $30 \mathrm{~s}$ and left to cure at room temperature for 3 hours. During curing some of the films are subjected to a homogeneous external magnetic field of $200 \mathrm{mT}$ produced by a cylindrical neodymium static magnet (diameter $\mathrm{D}=25 \mathrm{~mm}$, thickness $\mathrm{t}=10 \mathrm{~mm}$ ) and directed along the normal to the surface of the films. In order to achieve a complete curing, the samples were subsequently put in an oven at $80{ }^{\circ} \mathrm{C}$ overnight. The resulting films (thickness $400 \mu \mathrm{m}$ ) were then released from the Teflon mould and glued with silicone sealant (Elastosil, Wacher Chemie AG) on an epoxy substrate with a $10 \mathrm{~mm}$ central hole to allow the deflection of the free part of the film.

Following the above-mentioned procedure four different types of films were prepared: (a) Films with magnetic particles distributed homogeneously in the whole volume of the polymer (Samples $\mathrm{S}_{\mathrm{H}}$ ); (b) Films with magnetic particles whose easy axis is oriented preferentially in a direction normal to the surface, forming magnetic microchains throughout the polymer matrix 
(Samples $\mathrm{S}_{\mathrm{A}}$ ): this is obtained upon the application of an external magnetic field of $200 \mathrm{mT}$, as previously described; (c) Films $\mathrm{S}_{\mathrm{A}}$ subsequently magnetized in a $3 \mathrm{~T}$ magnetic field, higher than the coercivity of the particles (Samples $\mathrm{S}_{\mathrm{Apost3T}}$ ); (d) Films $\mathrm{S}_{\mathrm{H}}$ magnetized in a $3 \mathrm{~T}$ magnetic field, named $\mathrm{S}_{\mathrm{Hpost3T}}$.

The $3 \mathrm{~T}$ magnetization field was generated by a MRI Scanner (3 T Excite HDx GE Medical Systems): the magnetizing field direction was parallel to the aligning direction of the particles in order to achieve a synergistic effect. The samples were let under the $3 \mathrm{~T}$ magnetic field for 15 seconds.

2.3. Characterization. Morphological analysis: A stereomicroscope in transmission mode (Leica S8 APO) equipped with a digital camera accessory and image processing software was used to image the surface and the cross section of the magnetic films prepared according to the different, previously stated, methods.

SEM imaging was performed with a JEOL JSM-6490LA (Jeol-Japan), equipped with a tungsten thermionic electron source operated at $15 \mathrm{kV}$ of acceleration voltage. Samples were prepared via freeze fracture in liquid nitrogen to expose the cross section. The imaging for the $\mathrm{S}_{\mathrm{A}}$ and $\mathrm{S}_{\mathrm{H}}$ samples was performed using the backscattered electrons detector to have compositional contrast and to enhance the difference between the $\mathrm{Nd}_{2} \mathrm{Fe}_{14} \mathrm{~B}$ particles and the elastomeric matrix. Due to the non-conductive nature of the polymer, the low vacuum mode (25 Pa) was adopted in order to avoid metallic coating, which could have masked the compositional contrast.

Deflection measurements: The deflection was measured using a laser-optical sensor ILD 2200 (from Micro-Epsilon) and the schema of the experimental set-up is reported in Supporting Information (Figure S2). The magnetic films, previously fixed on an epoxy substrate with a central hole of $10 \mathrm{~mm}$ in diameter, deflect in a direction normal to their surface under the effect 
of an external magnetic field generated by a cylindrical neodymium static magnet (diameter $\mathrm{D}=$ $25 \mathrm{~mm}$, thickness $\mathrm{t}=10 \mathrm{~mm}$ ) and the sensor records the deflection value. The strength of the field is modified by varying the distance between the magnet and the film (Supporting Information Figure S3). The measurements were conducted three times on two different samples for each composition.

Magnetic characterization measurements: Measurements of static magnetization and hysteresis loops were performed on a Quantum Design MPMS SQUID (Superconducting Quantum Interference Device) magnetometer, equipped with a superconducting magnet capable of producing fields up to $7 \mathrm{~T}$. Hysteresis loops were recorded at room temperature with a maximum applied field of $\pm 3 \mathrm{~T}$. The applied field was normal to the surface of the films, so that its direction was parallel to the magnetic fields previously used during the alignment and magnetization procedures. The data were normalized with respect to the precise mass of the particles within the samples as defined by thermogravimetric analyses (TGA Q500-TA Instruments). Specifically, the samples used for the magnetic measurements and a pure elastomer sample were heated up to $900{ }^{\circ} \mathrm{C}$ at a rate of $10{ }^{\circ} \mathrm{C} \cdot \mathrm{min}^{-1}$ in air (flow rate $50 \mathrm{ml} \cdot \mathrm{min}^{-1}$ ), in order to degrade all the organic components. The residual mass obtained at the end of the experiment for each composite sample was compared with the residual mass obtained for the pure polymer in order to calculate the exact amount of magnetic content inside each film.

\section{Results and discussion:}

In order to evaluate the appropriate magnetic content of the composite films that leads to the highest magneto-responsivity, deflection measurements were first conducted on the $\mathrm{S}_{\mathrm{H}}$ composites loaded with magnetic particles amount up to $50 \mathrm{wt} \%$ (Figure 1). The displacement values increase with the rise of the particles concentration. In particular the maximum 
displacement achieved under an external magnetic field of $100 \mathrm{mT}$, is $41 \mu \mathrm{m}$ for the $30 \mathrm{wt} \% \mathrm{~S}_{\mathrm{H}}$ sample, $86 \%$ higher compared to the $22 \mu \mathrm{m}$ achieved for the $20 \mathrm{wt} \% \mathrm{~S}_{\mathrm{H}}$ sample. Further increase of the magnetic content does not induce any significant improvement on the deflection: the performance of the $50 \mathrm{wt} \%$ sample is similar to that of the 30 and $40 \mathrm{wt} \%$. This behaviour indicates that the deflection of the films is not solely a function of the magnetic particles content but also of the stiffness of the resulting composite film. In fact, the mechanical properties of the films are strictly dependent on both the amount of particles within the composite and its effect on the crosslinking process ${ }^{36,37}$ (Figure S4). The progressive addition of rigid particles within a soft polymeric matrix leads to a rise of the film's stiffness that hinders its deformation upon the effect of the external field even if the magnetic content is increased. ${ }^{12}$ Consequently, the right compromise between elastic properties and amount of particles should be identified for the optimum performance of the composite, and in the present case is revealed to be the $30 \mathrm{wt} \%$ one.

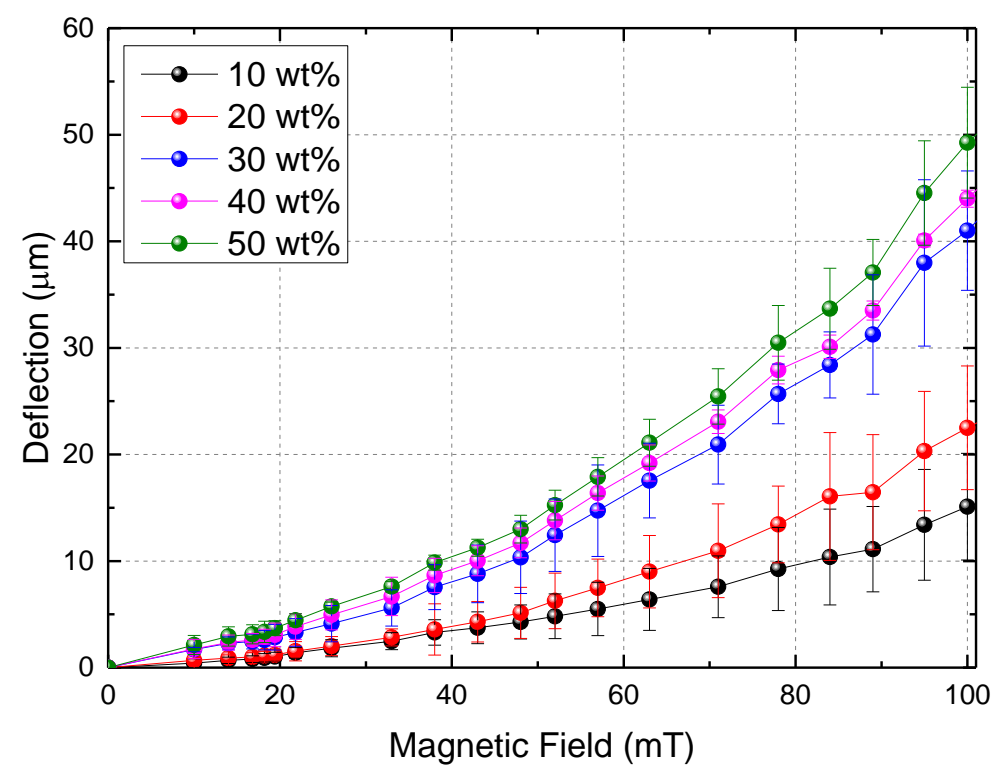

Figure 1. Deflection at different applied magnetic fields for $S_{H}$ films with different particles' concentration. 
To improve the deflection's performance of the selected sample, the alignment of the particles within the elastomeric matrix is achieved by the magnetophoretic transport and assembly process under an external magnetic field. In particular, $30 \mathrm{wt} \%$ of particles are mixed with the prepolymer and the curing of the composite is performed under an external magnetic field of 200 $\mathrm{mT}$. This drives the particles to assemble as microchains oriented parallel to the external field's direction and perpendicular to the surfaces of the films. ${ }^{29}$ The morphology of the cured films prepared without and with alignment of the magnetic particles, samples $\mathrm{S}_{\mathrm{H}}$ and $\mathrm{S}_{\mathrm{A}}$ respectively, presents significant differences as observed by optical microscopy (Figure 2). The top view of the films evidences that in the absence of alignment during curing $\left(\mathrm{S}_{\mathrm{H}}\right)$ the magnetic particles are homogeneously dispersed in the polymer matrix, which indicates the absence of relevant interactions between the particles (Figure 2a). However, when the particles are aligned during the polymer curing, they are assembled in a preferential direction forming organized features on the film's surface (Figure 2b). This is further confirmed by the investigation of the cross section of the two films (Figure 2c-d) where the formation of magnetic microchains throughout the thickness of the polymeric matrix is evidenced. This is mainly attributed to the interaction between the magnetic particles and the applied field. Specifically, during the alignment process the polymer is not yet cured, thus the magnetic particles can move freely in the viscous matrix under the effect of the external magnetic forces. Therefore, they tend to orient along the magnetic flux lines and to form localized microchains perpendicular to the films surfaces. After the end of the polymerization process, the microchains are permanently assembled into the elastomer matrix due to its high viscosity, which prevents any further movement of the particles. $^{29}$ 

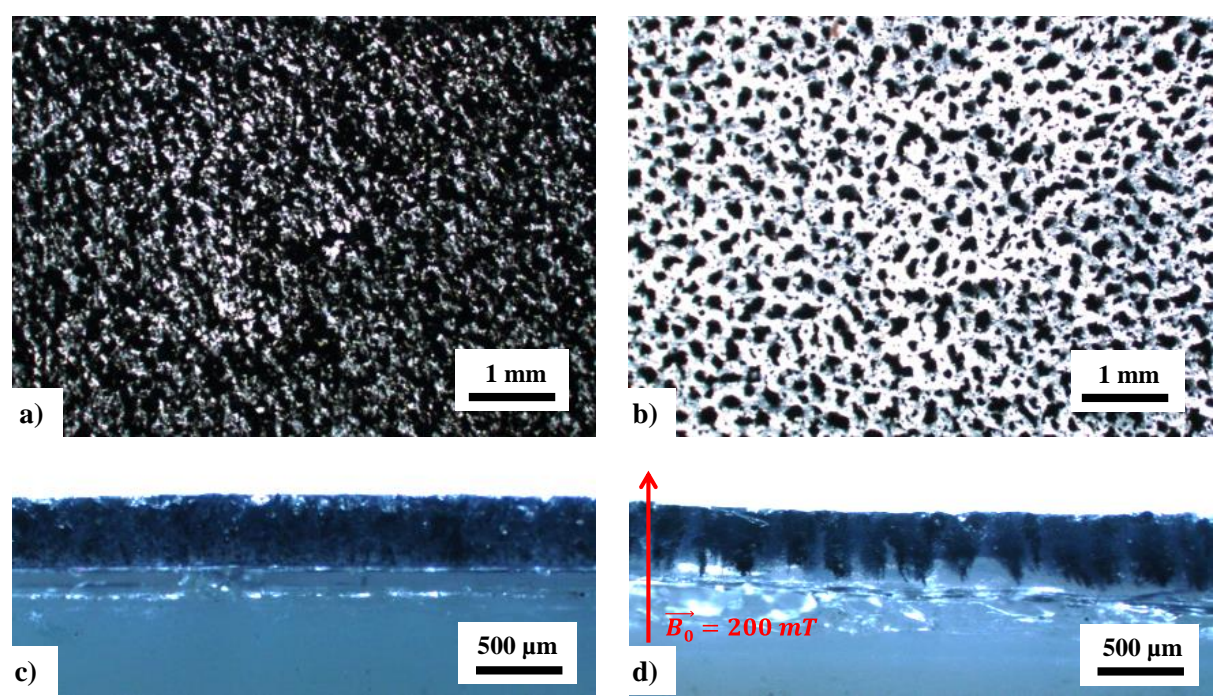

Figure 2. Optical microscopy images of magnetic films with $30 \mathrm{wt} \%$ magnetic particles distributed homogeneously throughout the surface and the volume of the film (a-c) or showing a preferential alignment vertically with respect to the film's surface (b-d). Top view (a-b) and cross section (c-d).

A deeper insight on the assembled microchains was achieved by SEM analysis as shown in Figure 3. In accordance to the optical microscopy study, the cross-section of the $\mathrm{S}_{\mathrm{H}}$ sample (Figure 3a) evidences randomly distributed magnetic particles within the whole polymer matrix. On the contrary, as presented in Figure 3b, the particles are distributed along a preferential direction at the $\mathrm{S}_{\mathrm{A}}$ film, and their composition is clearly shown by the EDX analysis presented in Supporting Information Figure S5. The formed microchains have a mean thickness of $130 \pm 35$ $\mu \mathrm{m}$, while the mean distance between them is $230 \pm 40 \mu \mathrm{m}$. 

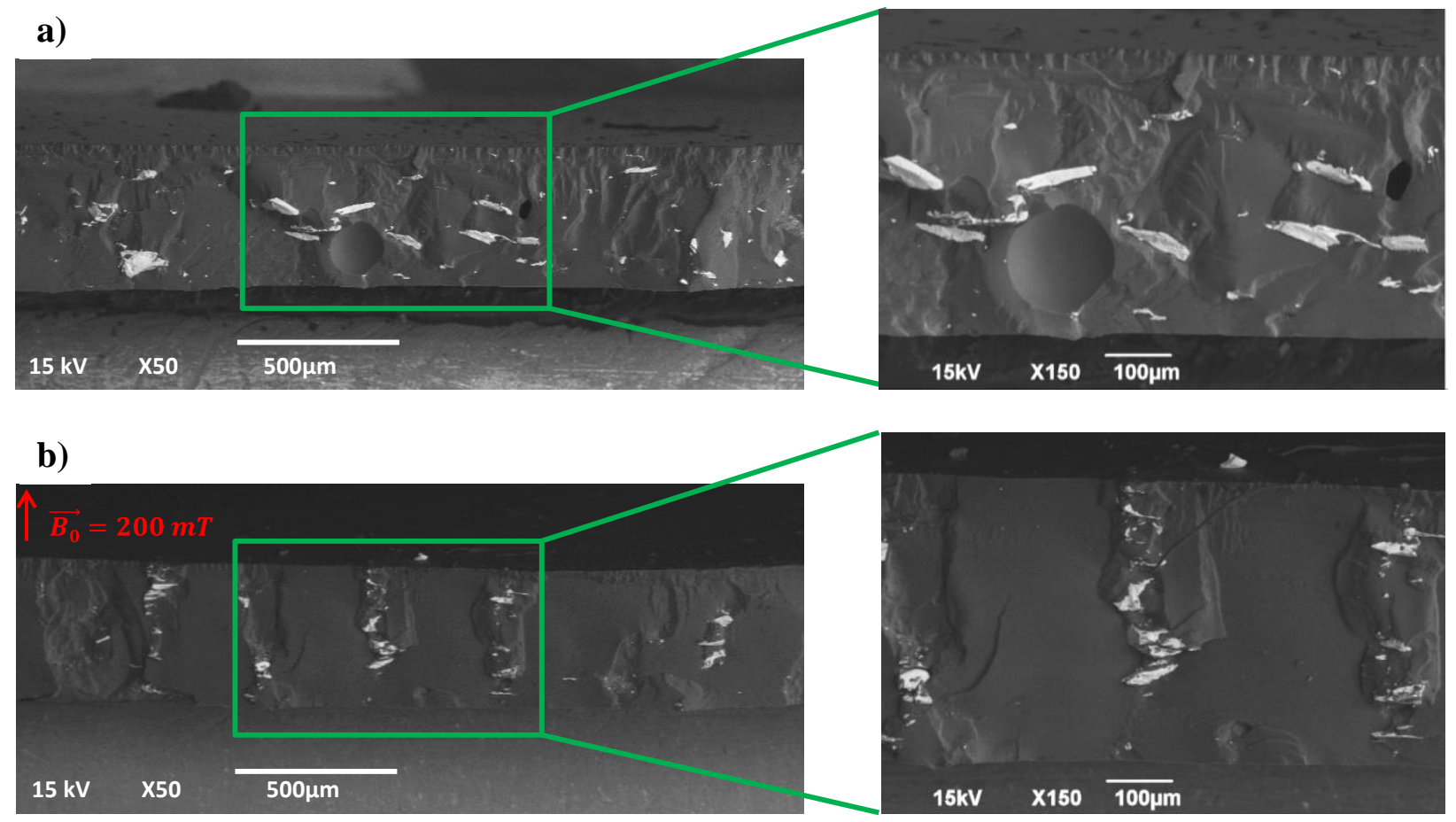

Figure 3. SEM images of a) $S_{H}$ and b) $S_{A}$ samples for both low and high magnifications. The loss of the particles along the assembled paths in (b) is attributed to the freeze fracture process.

The alignment of the particles during curing $\left(\mathrm{S}_{\mathrm{A}}\right)$ leads to a significant increase of the deflection with respect to the $S_{H}$ films as shown in Figure 4. In fact, since the magnetic field applied during the actuation is normal to the surface of the films, the magnetic microchains within the sample $\mathrm{S}_{\mathrm{A}}$ are parallel to it, and increase the composites' magneto-responsivity. In particular, the rough orientation of each particle's easy axis in the direction of the external field during the alignment process, in combination with their morphological arrangement in the form of microchains, result in the total increase of the magnetization of the $\mathrm{S}_{\mathrm{A}}$ film at the specific direction. $^{29}$ This enhancement is already effective at low magnetic fields (e.g. $42 \mathrm{mT}$ ) with an increment of $70 \%$ of the films' response (17 $\mu \mathrm{m}$ for $\mathrm{S}_{\mathrm{A}}$ against $10 \mu \mathrm{m}$ for $\left.\mathrm{S}_{\mathrm{H}}\right)$. Therefore, the significant increase in the deflection of the films after the alignment process indicates its importance for the great improvement of the magnetoresponsivity of the composite films. 


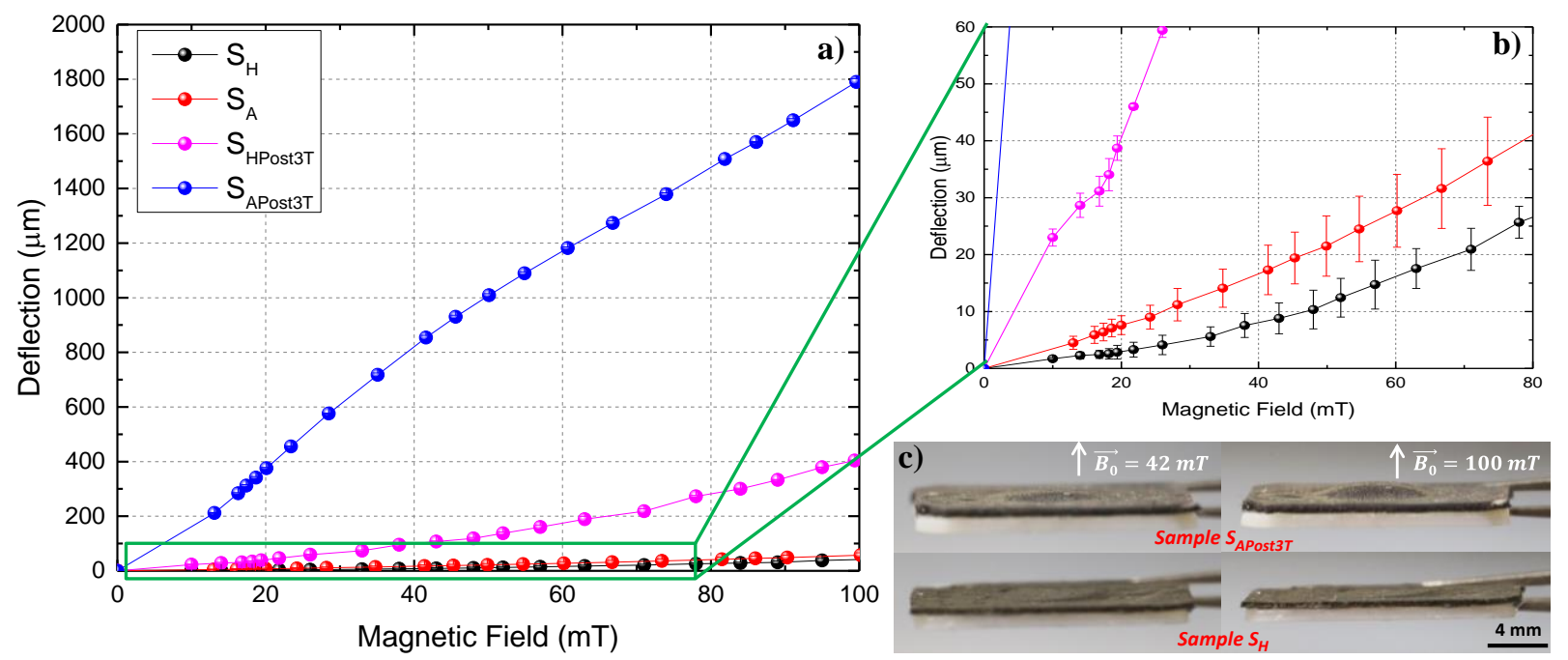

Figure 4. a) Deflection of the films containing $30 \mathrm{wt} \%$ magnetic particles under different magnetic fields (the error bars are smaller than the size of the symbols), b) magnification in low deflection region, c) photographs of the deflection of $\mathrm{S}_{\mathrm{AP} \text { sost3 }}$ (top) and $\mathrm{S}_{\mathrm{H}}$ (bottom) films under different magnetic fields.

The subsequent magnetization of the $\mathrm{S}_{\mathrm{H}}$ and $\mathrm{S}_{\mathrm{A}}$ films is conducted at a magnetic field of $3 \mathrm{~T}$, higher than the saturation magnetization of the particles $\left(\mathrm{M}_{\mathrm{sat}}=1250 \mathrm{mT}\right.$ as indicated by the supplier) with a direction normal to the films' surface. As reported in Figure 4, an impressive increase of the deflection of the magnetized film $\left(\mathrm{S}_{\mathrm{APost} 3 \mathrm{~T}}\right)$ is achieved with a maximum value of $1780 \mu \mathrm{m}$ for $100 \mathrm{mT}$, while at low fields the deflection is of hundreds of $\mu \mathrm{m}$, e.g. $850 \mu \mathrm{m}$ for 42 $\mathrm{mT}, 4900 \%$ more compared to the deflection recorded for the $\mathrm{S}_{\mathrm{A}}$ film and $8400 \%$ higher compared to the $\mathrm{S}_{\mathrm{H}}$ for the same actuation field. Indeed as shown in Figure $4 \mathrm{c}$ the deflection of the $\mathrm{S}_{\mathrm{AP} \text { ost3T }}$ is clearly visible, even at low magnetic fields $(42 \mathrm{mT})$, in contrary to the deflection of the $\mathrm{S}_{\mathrm{H}}$ sample. Interestingly, the enhancement of the magneto-responsivity due to the postmagnetization process is much more pronounced for the films where the particles are aligned in 
the form of microchains compared to the films where the particles are homogeneously dispersed, evidencing the importance of the alignment process before magnetization.

As already discussed, during the alignment process the particles assemble parallel to the external magnetic field, partially orienting their magnetic moment in that preferential direction and inducing a synergistic effect for the final magnetic moment of the assembled microchains. The subsequent magnetization of the films is a second important step for the impressive increase of the magneto-responsivity. In order to achieve the maximum performance, the applied magnetic field for the post-treatment needs to be higher than the one leading to saturation magnetization of the particles so as to assure that all the particles in the sample will align with the field, and to obtain a full magnetization. Indeed, magnetization performed with lower magnetic field $(500 \mathrm{mT})$ evidenced that the post-treatment was not sufficient to reach similar deflections with the $\mathrm{S}_{\mathrm{APost3T}}$ film (Supporting Information Figure $\mathrm{S6}$ ). Therefore, during the $\mathrm{S}_{\mathrm{AP} \text { ost3T }}$ film magnetization, the size of the magnetic domains is maximized as each magnetic particle reaches its saturation state, resulting in a subsequent increase of the total magnetic moment of the whole microchain of which is a component. In fact, the application of a high magnetic field promotes the increase of the residual magnetization of the ferromagnetic particles, which results in a higher responsivity with respect to the pristine ones. In this way, the total magnetic moment of the microchains (in the $\mathrm{S}_{\mathrm{APos} 3 \mathrm{~T}}$ film) is a synergistic effect of the magnetic moments of the individual particles while the magnetizing field in the post treatment provokes their complete rotation towards their preferential direction. This leads to highly efficient films and indeed, the deflection is much higher with respect to the $\mathrm{S}_{\mathrm{H}}$ films or to the films prepared with only one process (alignment or magnetization). 
The effect of the alignment and post-magnetization procedures on the magnetic performances of the films was confirmed by analysing their magnetic properties by collecting hysteresis loops at $298 \mathrm{~K}$. The initial magnetization curves (Figure 5a) show the magnetic response of the films when first exposed to an increasing field going from 0 to $3 \mathrm{~T}$, offering useful indications on their features. In particular, variations in the magnetization are clearly visible in the low-field region (up to $1 \mathrm{~T}$ ), where the $\mathrm{S}_{\mathrm{H}}$ and $\mathrm{S}_{\mathrm{APost3T}}$ films exhibit, respectively, the lowest and highest magnetization value. The effects of the alignment and magnetization are easily recognizable, with the first determining some domain wall movements (as should be expected due to the low field applied during alignment) resulting in a slight increase in the first magnetization curve of $\mathrm{S}_{\mathrm{A}}$ with respect to the curve of the $\mathrm{S}_{\mathrm{H}}$ film. On the other hand, the high field applied for the posttreatment magnetization determines large irreversible jumps of the domain walls along with rotation processes, which lead to the brisk increase in the first magnetization curves of the $\mathrm{S}_{\mathrm{HPost3T}}$ and $\mathrm{S}_{\mathrm{APos} 3 \mathrm{~T}}$ films with respect to $\mathrm{S}_{\mathrm{A}}$. The combination of both treatments works as a twostep process, with the alignment being responsible for the formation of microchains of particles with magnetic domains roughly oriented along a common direction and the magnetization maximising the size of the magnetic domains oriented along the magnetizing field.
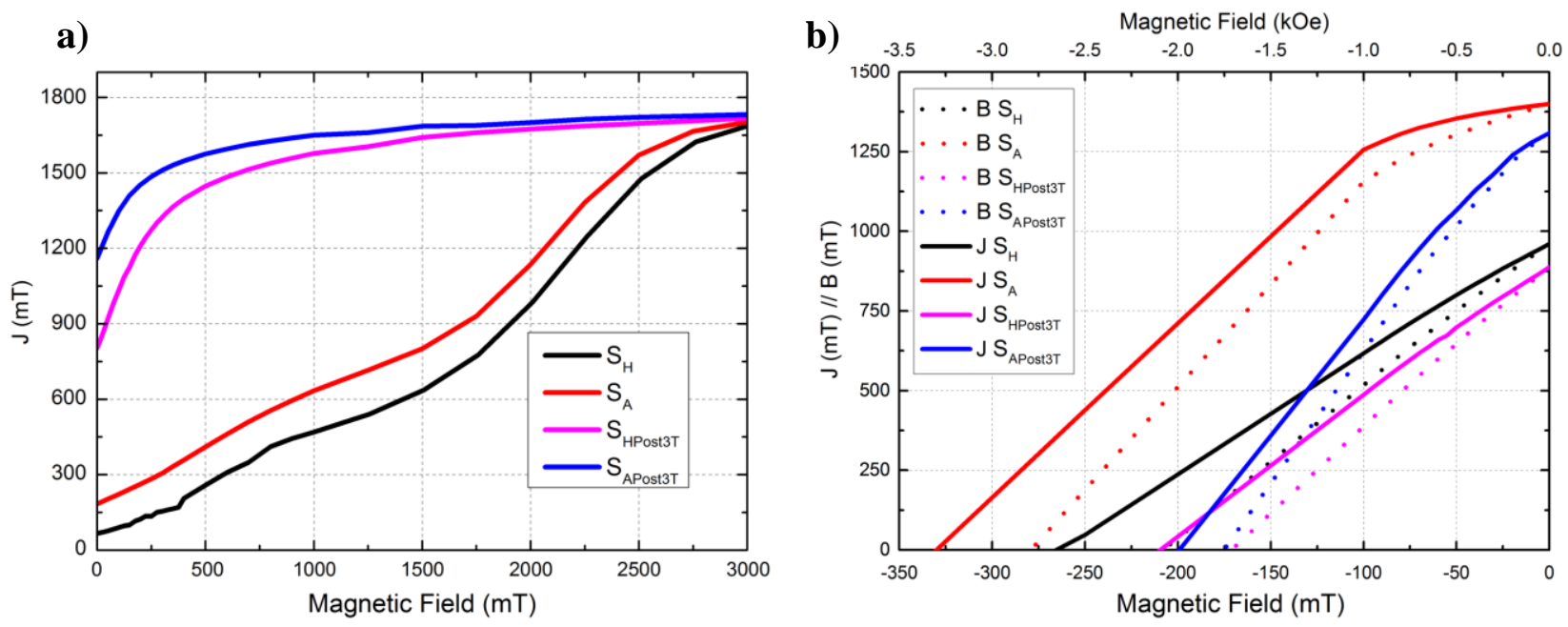
Figure 5. a) First magnetization curves from 0 up to $3 \mathrm{~T}$ recorded at $298 \mathrm{~K}$ for films containing $30 \mathrm{wt} \%$ magnetic particles; b) demagnetization curves of films containing $30 \mathrm{wt} \%$ magnetic particles after exposure to a $3 \mathrm{~T}$ external field. Full lines indicate magnetic polarization (J), dotted lines indicate magnetic flux density (B).

Given the high weight percentage of magnetic species present in the polymeric matrices, interactions should play a key role in varying their magnetic parameters. The role of interactions, the formation of different-sized magnetic domains and the overall variations occurring to the films, depending on the treatments of choice, can be studied more in detail by analysing the residual magnetization in absence of any external applied field (remanence, $\boldsymbol{B}_{\boldsymbol{r}}$ ) and the demagnetization field (coercivity $\boldsymbol{H}_{\boldsymbol{c}}$ ) (Figure $5 \mathrm{~b}$ and Table 1).

$\begin{array}{llllll}\text { Sample } & \text { Magnetic particles } & \boldsymbol{S}_{\boldsymbol{H}} & \boldsymbol{S}_{\text {HPost3T }} & \boldsymbol{S}_{\boldsymbol{A}} & \boldsymbol{S}_{\boldsymbol{A P o s t 3 T}} \\ \mathbf{B}_{\mathbf{r}}(\mathbf{m T}) & 1240 & 976 & 888 & 1400 & 1309 \\ \mathbf{H}_{\mathrm{ci}}(\mathbf{k O e}) & 18.0 & 2.7 & 2.1 & 3.3 & 2.0\end{array}$

Table 1. Magnetic parameters of parent particles and films.

The $\boldsymbol{B}_{\boldsymbol{r}}$ enhancement of the $\mathrm{S}_{\mathrm{A}}$ and $\mathrm{S}_{\mathrm{APost} 3 \mathrm{~T}}$ films and its decrease in $\mathrm{S}_{\mathrm{H}}$ and $\mathrm{S}_{\mathrm{HPost} 3 \mathrm{~T}}$ films with respect to the parent particles should be ascribed to the effects of the aligning process. The proximity of the particles assembled in the microchains favors exchange coupling, which in remanent state results in preserving an enhanced net magnetization. On the other hand, the absence of alignment in the preparation of $\mathrm{S}_{\mathrm{H}}$ and $\mathrm{S}_{\mathrm{HPost} 3 \mathrm{~T}}$ films determines the presence of spatially dispersed particles, so that only dipole-dipole interactions should be expected, which leads to a decreased remanence with respect to the parent particles. Moreover, another effect due to post-treatment magnetization can be observed with regards to remanence of both aligned and non-aligned samples. The decrease observed in the remanence of $3 \mathrm{~T}$ treated samples with 
respect to the non-treated ones suggests that the formation of bigger magnetic domains due to major movements of the domains walls works against maintaining the magnetic alignment of the samples in absence of an external field.

As expected, $\boldsymbol{H}_{c i}$ values of the films are systematically smaller than the parent particles one, since the high fraction of magnetic particles inside the films leads to interactions playing a stronger role than anisotropy in determining the magnetic features of the films. The increased alignment of grains after the exposition to a $3 \mathrm{~T}$ external field during the hysteresis, results in a

strong decrease in $\boldsymbol{H}_{\boldsymbol{c}}$, similar to what observed in $\mathrm{Nd}_{2} \mathrm{Fe}_{14} \mathrm{~B}$ sintered magnets. ${ }^{9,38,39}$ Once again the features of the samples vary depending on the post-magnetization process, with the $\mathrm{S}_{\mathrm{HPost} 3 \mathrm{~T}}$ and $\mathrm{S}_{\mathrm{APost3T}}$ samples showing the lowest $\boldsymbol{H}_{\boldsymbol{c} i}$ values with respect to the $\mathrm{S}_{\mathrm{H}}$ and $\mathrm{S}_{\mathrm{A}}$ samples. This suggests that the increase in size of the magnetic domains in the post-magnetization samples plays a role in further decreasing the $\boldsymbol{H}_{\boldsymbol{c} \boldsymbol{i}}$.

\section{Conclusions:}

This study clearly demonstrates the importance of both alignment of the $\mathrm{Nd}_{2} \mathrm{Fe}_{14} \mathrm{~B}$ magnetic particles and subsequent magnetization of the elastomeric composite in order to reach high deflection performances of films under magnetic actuation. The use of an external magnetic field for the magnetophoretic transport and assembly of the magnetic particles during the polymeric matrix's curing induces the formation of aligned magnetic microchains throughout the film's thickness. This results in an improvement of the film's deflection compared to the films where particles of same concentration are homogeneously dispersed. The subsequent magnetization of the films at a magnetic field higher than the critical field for saturation magnetization of the particles provokes the impressive increase of the deflection, with performances reaching deflections' values 85 times higher than those of non-treated films, with maximum deflection of 
$850 \mu \mathrm{m}$ at $42 \mathrm{mT}\left(10 \mu \mathrm{m}\right.$ for the $\mathrm{S}_{\mathrm{H}}$ film $)$ while at higher fields, e.g. $100 \mathrm{mT}$, the displacement is around $1780 \mu \mathrm{m}$. Magnetic studies confirm that the alignment process is responsible for the collective effect on the magnetic moment, increasing thus the magnetic response at the direction of the microchains while the post magnetization process results in the enhancement of the magnetic domains sizes that are oriented along the magnetizing field. These films are suitable for applications in which high deformations at low magnetic fields are requested.

\section{ASSOCIATED CONTENT}

Supporting Information. SEM images and EDX mapping of the magnetic particles, experimental set-up for the measurements of film's deflection; magnetic field gradient used for the deflection experiments; tensile tests of the silicone composites, SEM images and EDX mapping of the $\mathrm{S}_{\mathrm{A}}$ sample, deflection measurements under diverse magnetic fields for $500 \mathrm{mT}$ post-magnetization field. The Supporting Information is available free of charge on the ACS Publications website at http://pubs.acs.org.

\section{AUTHOR INFORMATION}

\section{Corresponding Authors}

*E-mail: sophie.marchi@iit.it, despina.fragouli@iit.it

\section{Funding Sources}

This work is financed by the EU within the BLINDPAD Project (FP7/2007-2013 Grant Agreement No. 611621). 


\section{ACKNOWLEDGMENT}

The magnetization at $3 \mathrm{~T}$ was kindly achieved by Francesca Frijia in U.O.C. Bioingegneria e ingegneria clinica (CNR-Regione Toscana) Fondazione "G. Monasterio" CNR-Regione Toscana Area della Ricerca S. Cataldo. Simone Lauciello and Alice Scarpellini from Nanochemistry, and Lara Marini from Nanophysics, Istituto Italiano di Tecnologia, are gratefully acknowledged for SEM/EDX and TGA analyses respectively.

\section{References:}

(1) Koch, M.; Evans, A.G.R.; Brunnschweiller, A. The Dynamic Micropump Driven with a Screen Printed PZT Actuator. J. Micromech. Microeng. 1998, 8, 119-122.

(2) Chan, W.S.; Saarinen, M.J.; Talghader J.J. Fabrication and Operation of an Electrostatic Actuator for Controlling Nanometer-Scale Gaps in Collapsed Cantilever Heterostructures. Appl. Phys. Lett. 2013, 102, 243508-1-3.

(3) Amakawa, H.; Fukuzawa, K.; Shikida, M.; Tsuji, H.; Zhang, H.; Itoh, S. An Electrostatic Actuator for Dual-Axis Micro-Mechanical Probe on Friction Force Microscope. Sensor Actuat. A-Phys. 2012, 175, 94-100.

(4) Chunder, A.; Etcheverry, K.; Londe, G.; Cho, H.J.; Zhai, L. Conformal Switchable Superhydrophobic/hydrophilic Surfaces for Microscale Flow Control. Colloid Surface A 2009, $333,187-193$.

(5) Neagu, C.R.; Gardeniers, J.G.E.; Elwenspoek, M.; Kelly, J.J. An Electrochemical Microactuator: Principle and First Results. J. Microelectromech. Syst. 1996, 5, 2-9. 
(6) Tice, J.D.; Bassett, T.A.; Desai, A.V.; Apblett, C.A.; Kenis, P.J.A. A Monolithic Poly(Dimethylsiloxane) Electrostatic Actuator for Controlling Integrated Pneumatic Microsystems. Sensor Actuat. A-Phys. 2013, 196, 22-29.

(7) Pabst, O.; Hölzer, S.; Beckert, E.; Perelaer, J.; Schubert, U.S.; Eberhardt, R.; Tünnermann. Inkjet Printed Micropump Actuator Based on Piezoelectric Polymers: Device Performance and Morphology Studies. Org. Electron. 2014, 15, 3306-3315.

(8) Khoo M.; Liu, C.; Micro Magnetic Silicone Elastomer Membrane Actuator. Sensor Actuat. A-Phys. 2001, 89, 259-266.

(9) Wang, W.S.; Yao, Z.M.; Chen, J.C.; Fang, J. Composite Elastic Magnet Films with Hard Magnetic Feature. J. Micromech. Microeng. 2004, 14, 1321-1327.

(10) Fahrni, F.; Prins, M.W.J.; van IJzendoorn, L.J. Magnetization and Actuation of Polymeric Microstructure with Magnetic Nanoparticles for Application in Microfluidics. J. Magn. Magn. Mater. 2009, 321, 1843-1850.

(11) Pirmoradi, F.N.; Cheng, L.; Chiao, M. A Magnetic Poly(Dimethylsiloxane) Composite Membrane Incorporated with Uniformly Dispersed, Coated Iron Oxide Nanoparticles. J. Micromech. Microeng. 2010, 20, 015032-1-7.

(12) Li, J.; Zhang, M.; Wang, L.; Li, W. Sheng, P.; Wen, W. Design and Fabrication of Microfluidic Mixer from Carbonyl Iron-PDMS Composite Membrane. Microfluid. Nanofluid. 2011, 10, 919-925.

(13) Thévenot, J.; Oliveira, H.; Sandre, O.; Lecommandoux, S. Magnetic Responsive Polymer Composite Materials. Chem. Soc. Rev. 2013, 42, 7099-7116. 
(14) Richter, A.; Klatt, S.; Paschew, G.; Klenke, C.; Micropumps Operated by Swelling and Shrinking of Temperature-Sensitive Hydrogels. Lab Chip 2009, 9, 613-618.

(15) Diller, E.; Miyashita, S.; Sitti, M. Remotely Addressable Magnetic Composite Micropumps. RSC Adv. 2012, 2, 3850-3856.

(16) Tice, J.D.; Desai, A.V.; Bassett, T.A.; Apblett, C.A.; Kenis, P.J.A. Control of PressureDriven Components in Integrated Microfluidic Devices Using an On-Chip Electrostatic Microvalve. RSC Adv. 2014, 4, 51593-51602.

(17) Olfatnia, M.; Cui L.; Chopra P.; Awtar S. Large Range Dual-Axis Micro-Stage Driven by Electrostatic Comb-Drive Actuators. J. Micromech. Microeng. 2013, 23, 105008-1-11.

(18) Zhang, X.; Pint, C.L.; Lee, M.H.; Schubert, B.E.; Jamshidi, A.; Takei, K.; Ko, H.; Gillies, A.; Bardhan, R.; Urban, J.J.; Wu, M.; Fearing, R.; Javey, A. Optically- and ThermallyResponsive Programmable Materials Based on Carbon Nanotube-Hydrogel Polymer Composites. Nano Lett. 2011, 11, 3239-3244.

(19) Changhai, R.; Lining, S. Hysteresis and Creep Compensation for Piezoelectric Actuator in Open-Loop Operation. Sensor Actuat. A-Phys. 2005, 122, 124-130.

(20) Zrìnyi, M. In Smart Polymers and Their Applications; Aguilar, M.R.; Roman, J.S., Eds.; Woodhead Publishing, 2014; Chapter 5, pp 134-165.

(21) Fahrni, F.; Prins M.W.J.; van IJzendoorn, L.J.; Micro-Fluidic Actuation Using Magnetic Artificial Cilia. Lab Chip, 2009, 9, 3413-3421. 
(22) Nanni, G.; Petroni, S.; Fragouli, D.; Amato, M.; De Vittorio, M.; Athanassiou, A. Microfabrication of Magnetically Actuated PDMS-Iron Composite Membranes. Microelectron. Eng. 2012, 98, 607-609.

(23) Abramchuk, S.; Kramarenko, E.; Stepanov, G.; Nikitin, L.V.; Filipcsei, G.; Khokhlov, A.R.; Zrìnyi, M. Novel Highly Elastic Magnetic Materials for Dampers and Seals: Part I. Preparation and Characterization of the Elastic Materials. Polym. Adv. Technol. 2007, 18, 883890.

(24) Peng, S.; Zhang, M.; Niu, X.; Wen, W.; Sheng, P.; Liu, Z.; Shi, J. Magnetically Responsive Elastic Microspheres. Appl. Phys. Lett. 2008, 92, 012108-1-3.

(25) Abramchuk, S.; Kramarenko, E.; Grishin, D.; Stepanov, G.; Nikitin, L.V.; Filipcsei, G.; Khokhlov, A.R.; Zrìnyi, M. Novel Highly Elastic Magnetic Materials for Dampers and Seals: Part II. Material Behavior in a Magnetic Field. Polym. Adv. Technol. 2007, 18, 883-890.

(26) Singh, A.; Shirolkar, M.; Limaye, M.V.; Gokhale, S.; Khan-Malek, C.; Kulkarni, S.K. A Magnetic Nano-Composite Soft Polymeric Membrane. Microsyst. Technol. 2013, 19, 409-418.

(27) Ormerod, J.; Constantinides, S. Bonded Permanent Magnets: Current Status and Future Opportunities. J. Appl. Phys. 1997, 81, 4816-4820.

(28) Panchanathan, V. Magnequench Magnets Status Overview. J. Mater. Eng. Perform. 1995, $4,423-429$.

(29) Fragouli, D.; Buonsanti, R.; Bertoni, G.; Sangregorio, C.; Innocenti, C.; Falqui, A.; Gatteschi, D.; Cozzoli, P.D.; Athanassiou, A.; Cingolani, R. Dynamical Formation of Spatially 
Localized Arrays of Aligned Nanowires in Plastic Films with Magnetic Anisotropy. ACS Nano 2010, 4, 1873-1878.

(30) Lorenzo, D.; Fragouli, D.; Bertoni, G.; Innocenti, C.; Anyfantis, G.C.; Cozzoli, P.D.; Cingolani, R.; Athanassiou, A. Formation and Magnetic Manipulation of Periodically Aligned Microchains in Thin Plastic Membranes. J. Appl. Phys. 2012, 112, 083927-1-8.

(31) Fragouli, D.; Torre, B.; Villafiorita-Monteleone, F.; Kostopoulou, A.; Nanni, G.; Falqui, A.; Casu, A.; Lappas, A.; Cingolani, R.; Athanassiou, A. Nanocomposite Pattern-Mediated Magnetic Interactions for Localized Deposition of Nanomaterials. ACS Appl. Mater. Interfaces 2013, $5,7253-7257$.

(32) Fragouli, D.; Das, A.; Innocenti, C.; Guttikonda, Y.; Rahman, S.; Liu, L.; Caramia, V.; Megaridis, C.M.; Athanassiou, A. Polymeric Films with Electric and Magnetic Anisotropy Due to Magnetically Assembled Functional Nanofibers. ACS Appl. Mater. Interfaces 2014, 6, 453541.

(33) Ghosh, S.; Puri, I.K. Soft Polymer Magnetic Nanocomposites: Microstructure Patterning by Magnetophoretic Transport and Self-Assembly. Soft Matter 2013, 9, 2024-2029.

(34) Robbes, A.S.; Cousin, F.; Meneau, F.; Dalmas, F.; Boué, F.; Jestin, J. Nanocomposite Materials with Controlled Anisotropic Reinforcement Triggered by Magnetic Self-Assembly. Macromolecules 2011, 44, 8858-8865.

(35) Kim, J.; Chung, S.E.; Choi, S.E.; Lee, H.; Kim, J.; Kwon, S. Programming Magnetic Anisotropy in Polymeric Microactuators. Nat. Mater. 2011, DOI: 10.1038/nmat3090. 
(36) Roppolo, I.; Shahzad, N.; Sacco, A.; Tresso, E. Sangermano, M. Multifunctional NIRReflective and Self-Cleaning UV-Cured Coating for Solar Cell Applications Based on Cycloaliphatic Epoxy Resin. Prog. Org. Coat. 2014, 77, 458-462.

(37) Marchi, S.; Sangermano, M.; Meier, P.; Kornmann, X. Preparation and Characterization of PDMS Composites for Outdoor Polymeric Insulators. Polym. Composite 2014, 35, 1253-1262.

(38) Matsuura, Y.; Hoshijima J.; Ishii, R. Relation Between $\mathrm{Nd}_{2} \mathrm{Fe}_{14} \mathrm{~B}$ Grain Alignment and Coercive Force Decrease Ratio in NdFeB Sintered Magnets. J. Magn. Magn. Mater. 2013, 336, 88-92.

(39) Herbst, J.F. $\mathrm{R}_{2} \mathrm{Fe}_{14} \mathrm{~B}$ Materials: Intrinsic Properties and Technological Aspects. Rev. Mod. Phys. 1991, 63, 819-898.

Table Of Content Graphic

The magnetophoretic transport of magnetic particles during the curing of the polymeric matrix allows their assembly in aligned microchains. The combination of this method with a subsequent magnetization at high magnetic field of the resulting composite induces a remarkable increase of the film's responsivity upon the effect of an external magnetic field with respect to a homogeneous dispersion of magnetic particles within the polymer.

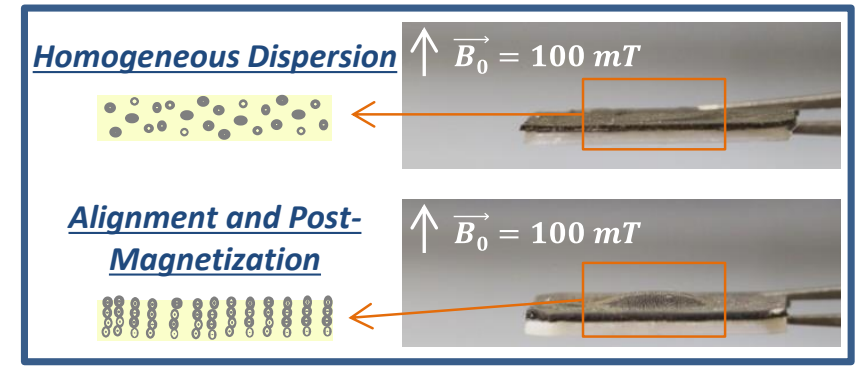

